\title{
Quantification of Cytoskeletal Protein Localization from High-Content Images
}

\author{
Shiwen Zhu ${ }^{1}$, Paul Matsudaira ${ }^{1,2}$, Roy Welsch ${ }^{1,4}$, and Jagath C. Rajapakse ${ }^{1,3,5}$ \\ ${ }^{1}$ Computation and System Biology, Singapore-MIT Alliance, Nanyang Technological \\ University, Singapore 637460 \\ ${ }^{2}$ Department of Biology Science, National University of Singapore, Singapore 117543 \\ ${ }^{3}$ School of Computer Engineering, Nanyang Technological University, Singapore 639798 \\ ${ }^{4}$ Sloan School of Management, Massachusetts Institute of Technology, Cambridge, \\ Massachusetts 02142, USA \\ ${ }^{5}$ Department of Biological Engineering, Massachusetts Institute of Technology, Cambridge, \\ Massachusetts 02139, USA
}

\begin{abstract}
Cytoskeletal proteins function as dynamic and complex components in many aspects of cell physiology and the maintenance of cell structure. However, very little is known about the coordinated system of these proteins. The knowledge of subcellular localization of proteins is crucial for understanding how proteins function within a cell. We present a framework for quantification of cytoskeletal protein localization from high-content microscopic images. Analyses of high content images of cells transfected by cytoskeleton genes involve individual cell segmentation, intensity transformation of subcellular compartments, protein segmentation based on correlation coefficients, and colocalization quantification of proteins in subcellular components. By quantifying the abundance of proteins in different compartments, we generate colocalization profiles that give insights into the functions of different cytoskeletal proteins.
\end{abstract}

Keywords: Colocalization, cytoskeletal proteins, subcellular localization, cytoskeleton.

\section{Introduction}

The cytoskeleton is a cellular skeleton - a dynamic structure in all eukaryotic cells and some of prokaryotic cells - that function dynamically in many aspects including the maintenance of cell shape, the protection of cells, the organization of the cytoplasm, the support of the cellular machinery for motility, the transportation, the organization of cells into tissues and the signaling. Since cytoskeletal proteins are involved in so many functions, they are chemically connected to the reactions of metabolism and to the complex functional networks of small molecules and enzymes that transport signals within cells [1][2]. With those signals, cytoskeletal proteins generate harmonious responses to the coordinated efforts of cellular networks. However, very little is known about the coordinated system of these proteins. Investigation of the exact roles of cytoskeletal proteins, therefore, has become an 
important task that would greatly benefit many research areas including cellular mechanics, subcellular organization, metabolic signaling pathway modeling, early development of cancer, etc.

The knowledge of subcellular location of proteins is crucial for understanding how proteins function within a cell. Fluorescent microscopy has been used more and more frequently to identify protein subcellular locations through image processing, feature extraction, and pattern recognition [3][4]. Machine learning methods have been previously applied for identifying and predicting the localization patterns of proteins by using training data [5][6][7][8]. But such methods were intended for single cell and single channel data.

How different proteins interact with more than one subcellular compartment and their presence in more than one location has not been addressed. More cellular compartments need to be considered as predicting candidates for a protein's location and prediction of a single subcellular protein pattern is not sufficient. Thus we chose to focus on fluorescent signal colocalization - a measurement of overlap between two signals. Quantifying a single colocalization parameter is necessary so that a variety of proteins can be quickly and easily compared without bias. Determining the colocalization between cytoskeletal proteins and subcellular components-such as nucleus, cytoplasm, plasma membrane, actin network, etc., will help to define and simplify the proteins' operations and locations.

There are two basic ways to measure the colocalization [9]: global statistical approaches that perform intensity correlation coefficient based analyses; and objectbased approaches. The global statistical approaches mainly use statistics to assess the relationship between fluorescence intensities in different compartments, including Pearson's coefficient [10], overlap coefficient [10], a statistical significance algorithm based on Pearson's coefficient [11], intensity correlation analysis [12], etc. However, the global statistical approaches rely on individual pixel coincidence analysis, globally providing colocalization estimation of the whole image but not of a unique structure. In the situation of low transfection efficiency, the cells with no or less GFP signals will pull down the overall colocalization estimates. Several methods of objectbased approaches have been proposed such as comparing the position of the centroids or intensity centers of the objects [13] and normalized mean deviation product [14]. But they all focus on protein-protein colocalization analysis, which is the correlation between two different protein channels. In the protein subcellular colocalization analysis, the intensities within subcellular compartments are not a major concern. For example, DNA is stained to represent the nucleus. The DNA intensities in nuclear compartment may not be uniform, but our major concern is how the protein is colocalized in the nucleus. Therefore, we introduce a new colocalization measurement inducing colocalization profiles indicating the amount of colocalization of proteins and subcellular compartments.

Experiments were carried out on HeLa cell lines transfected with cytoskeletal protein genes. High content images of protein localization were measured and clolocalization profiles were generated. Statistical analysis showed that the cytoskeletal proteins can be clustered into several groups with similar colocalization patterns. 


\section{Method}

In order to quantify subcellular localization of cytoskeletal proteins with a single parameter, the colocalization, we developed a computational framework involving individual cell segmentation, protein segmentation, intensity transformation of subcellular compartments, and colocalization computing. In what follows, we describe the different steps involved in our approach.

\subsection{Cell Segmentation}

Images were segmented into small objects using a multi-resolution segmentation technique based on object-oriented image analysis. This method is used to create object primitives as the first processing step in the segmentation analysis. The criterion for the segmentation is that average heterogeneity of image objects weighted by their size in pixels should be minimized. After the primary segmentation, the image objects are classified as nuclear objects and cell body objects based on flexible thresholds of nuclei and cell intensities. The nucleus objects are used as seeds for region growing method for cell segmentation, on the assumption that each cell has only one nucleus. A rule set was then developed for cell segmentation. The cell body object was fused with its neighbor nucleus. When one cell body object has more than one neighbor nuclear object, it was fused with the nuclear object that shared the largest border with it. The region growing method with multi-resolution segmentation provided better segmentation results than other advanced segmentation algorithms (Fig. 1): Level set method with shape marker and marking function [15] combined with nuclear information and level set method with topological dependence [16].

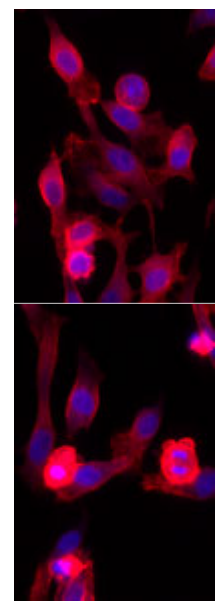

(a)

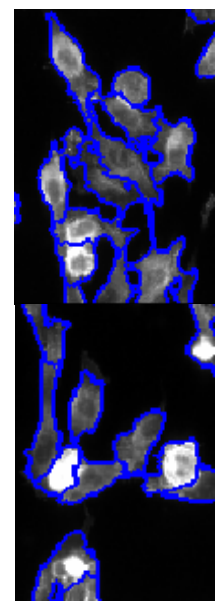

(b)

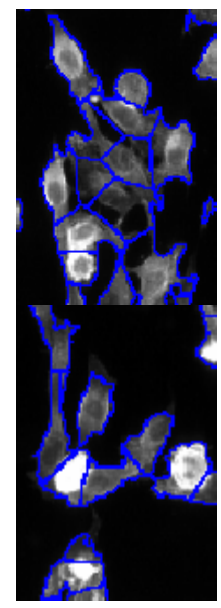

(c)

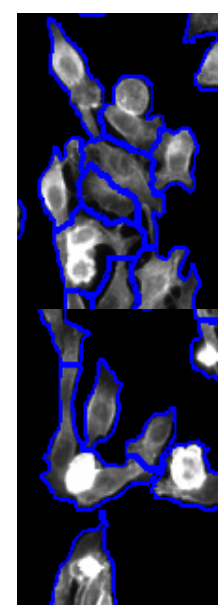

(d)

Fig. 1. (a) Images of actin and nuclear channels, and segmentation results of (b) region growing with multi-resolution segmentation, (c) adaptive level set method with shape marker combined with nuclear information, and (d) level set method with topological dependence 
In the experiments, cells were labeled with fluorescence to highlight nuclei, actin, and proteins of the cells. The green fluoresce protein (GFP) was selected to identify a particular protein. The nuclear and actin channels were used to identify subcellular compartments. Let $f_{n}: \Omega \rightarrow R, f_{a}: \Omega \rightarrow R$ and $f_{g}: \Omega \rightarrow R$ represent the images of nuclear channel, the actin channel, and the GFP channel, respectively, where $\Omega \subset \mathrm{R}^{2}$ is the $2 \mathrm{D}$ image domain and $x \in \Omega$ denotes the 2-D coordinates of a pixel site in the image. Let $w_{i} \subset \Omega$ denote the area of an individual cell, and $i \in N$ be the labels of cells in one image.

\subsection{Intensity Transformation of Subcellular Compartments}

In this study, we are interested in five subcellular compartments: nucleus, cytoplasm, actin, plasma membrane, and cytosol. For most of the subcellular compartments, the intensity distributions of labeled signals were uniform, but the colocalization amounts of proteins were different. Thus the intensity transformations of subcellular compartments are performed using an intensity information of actin and nucleus staining as well as the position and relation information of subcellular compartments. Instead of computing the colocalization of the protein signal and the compartment signal, we compute the colocalization of the protein signal and the intensity transformed images of the compartments. Let a compartment be denoted by $c=\{$ nucleus, cytoplasm, actin, membrane, cytosol $\}$, and $\tilde{f}_{c}: \Omega \rightarrow R$ denote the intensity transformed image in the compartment $c$.

Actin: This is the compartment identified from the actin channel. A ceiling threshold is used to account for over-saturation and the intensity values in the actin channel were rescaled to the range $[0,1]$.

$$
\tilde{f}_{\text {actin }}(x)=\left\{\begin{array}{c}
1, \quad f_{a}(x) \geq t_{a} ; \\
\frac{\left(f_{a}(x)-t_{a}^{\prime}\right)}{\left(t_{a}-t_{a}^{\prime}\right)}, \quad t_{a}>f_{a}(x) \geq t_{a}^{\prime} ; \quad x \in w_{i} ; \\
0, \quad f_{a}(x)<t_{a}^{\prime} .
\end{array}\right.
$$

where $t_{a}$ and $t_{a}^{\prime}$ are positive numbers representing upper and lower thresholds of the actin channel.

Cytoplasm: This is the compartment identified as the non-nuclear region. The transformation for the cytoplasm compartment is kept uniform.

$$
\tilde{f}_{\text {cytosplasm }}(x)=1-\tilde{f}_{\text {nucleus }}(x), \quad x \in w_{i} ;
$$


Nucleus: This is the compartment identified from the nuclei channel. We keep the intensities of the nucleus compartment uniform:

$$
\tilde{f}_{\text {nucleus }}(x)=\left\{\begin{array}{ll}
1, & f_{n}(x) \geq t_{n} \\
0, & f_{n}(x)<t_{n}
\end{array}, \quad x \in w_{i} ;\right.
$$

$t_{n}$ represents the threshold of the nuclei channel.

Plasma Membrane: This is the compartment identified as the border region of a cell. The intensities of plasma membrane were transformed using an exponential function of the minimum Euclidean distance to the cell border.

$$
\tilde{f}_{\text {membrane }}(x)=t_{m} \cdot \exp \left[-\left(\frac{\left(d(x)-t_{m}^{\prime}\right)}{t_{m}^{\prime \prime}}\right)^{2}\right], \quad x \in w_{i} ;
$$

$$
\text { Where } \quad d(x)=\min \sqrt{\left(x-x^{\prime}\right)^{2}}, \quad x \in w_{i}, x^{\prime} \in \partial w_{i} \text {; }
$$

and $t_{m}, t_{m}^{\prime}, t_{m}^{\prime \prime}$ are positive numbers representing the parameters of the exponential function with $\partial w_{i}$ representing the border.

Cytosol: This is the compartment identified as cytoplasm without the components of actin and the plasma membrane.

$$
\tilde{f}_{\text {cytosol }}(x)=\max \left\{0, \tilde{f}_{\text {cytoplasm }}(x)-\tilde{f}_{\text {membrane }}(x)-\tilde{f}_{\text {actin }}(x)\right\}, \quad x \in w_{i} ;
$$

\subsection{Protein Segmentation}

In order to identify the protein localization, cells were transfected with the protein tagged with GFP. By localizing the scattering of GFP-tagged proteins in the cells, its localizations in different subcellular compartments were identified. Before identifying the subcellular localization of the protein, the segmentation of protein needs to be correctly performed. Since the GFP intensities vary in different cells and compartments in different images, protein segmentation becomes a crucial component in finding the balance between capturing most of the protein information and highlighting the most specific protein information. Because the protein exists as small units, it cannot be segmented into one connected component. Thus, classical segmentation algorithms, such as watershed and region growing, become unsuitable. An automated thresholding method of identification of colocalized pixels has been earlier developed for protein-protein colocalization analysis [11].

Therefore, we develop an algorithm to segment the proteins by thresholding based on the correlation of its intensities with that of the responding compartment. The basic idea is to preserve most of the GFP pixels that are correlated with the intensities of the cell or its compartments, and remove the pixels that are least correlated or distributed almost randomly. 
For each candidate threshold, the correlation coefficient is computed on both selected (intensities higher than the candidate threshold) and unselected pixels (intensities lower than the candidate threshold). Correlation coefficients high on selected pixels and low on unselected pixels indicate that the current thresholds can save high correlated pixels and remove low correlated pixels, respectively. Thus, we want to achieve the proper balance between these two thresholds.

Several correlation coefficients were tested such as Pearson correlation coefficient and overlap coefficient. The intensity correlation quotient (ICQ) provided the best segmentation results. For one given cell image with channel $f_{1}, f_{2}$, and a given image region $w$, the ICQ value is based on the intensity correlation coefficient $\rho$ [17]. The correlation coefficient at a pixel is:

$$
\rho\left(f_{1}, f_{2}, x\right)=\left(f_{1}(x)-\mu_{1}\right)\left(f_{2}(x)-\mu_{2}\right), \quad x \in w ;
$$

$\mu_{1}$ and $\mu_{2}$ denote the mean intensities values with the region $w$ of the two channels.

The ICQ is defined as the ratio of the positively correlated pixels and the negatively correlated pixels in the region $w$. The correlation coefficient for two denoted areas is:

$$
\rho\left(f_{1}, f_{2}, w\right)=\frac{\delta^{+}}{\delta^{+}+\delta^{-}}-0.5
$$

where $\delta^{+}=\sum_{x \in w} \delta(\rho(x)>0)$ donates the total number of positively correlated pixels, and $\delta^{-}=\sum_{x \in w} \delta(\rho(x)<0)$ donates the total number of negatively correlated pixels. The range of ICQ falls between $\left[\begin{array}{cc}-0.5 & 0.5\end{array}\right]$. When ICQ $\approx 0$, random correlation; $-0.5 \leq \mathrm{ICQ}<0$, negative correlation; $0<\mathrm{ICQ} \leq 0.5$, positive correlation.

The Algorithm 1 gives a way to determine the optimum threshold for protein segmentation within the cell. $f_{\text {cell }}: \Omega \rightarrow R$ is the cell image obtained by combining the nuclei channel and the actin channel: $f_{\text {cell }}(x)=f_{a}(x)+f_{n}(x), x \in w_{i} . T$ is the final threshold generated for protein segmentation in this particular cell region $w_{i}$.

\subsection{Colocalization}

After segmentation of proteins within the cell and intensity transformation of subcellular compartments, the colocalization of proteins and subcellular compartments is quantified by a "colocalization" measurement that gives a better understanding about the percentage protein localized in a compartment. The colocalization of a protein $p$ in a compartment $c$ is defined as:

$$
\operatorname{Coloc}(c, p)=\frac{\sum_{x \in W_{p}} f_{\mathrm{p}}(x) \cdot \tilde{f}_{c}(x)}{\sum_{x \in W_{p}} f_{\mathrm{p}}(x)} ;
$$


where $W_{p}$ represents the set of pixels in the region occupied by the protein $p \cdot f_{\mathrm{p}}(x)$ is the intensity distribution of the GFP channel highlighting protein $p$ and $\tilde{f}_{c}(x)$ is the intensity transformation of the compartment $c$.

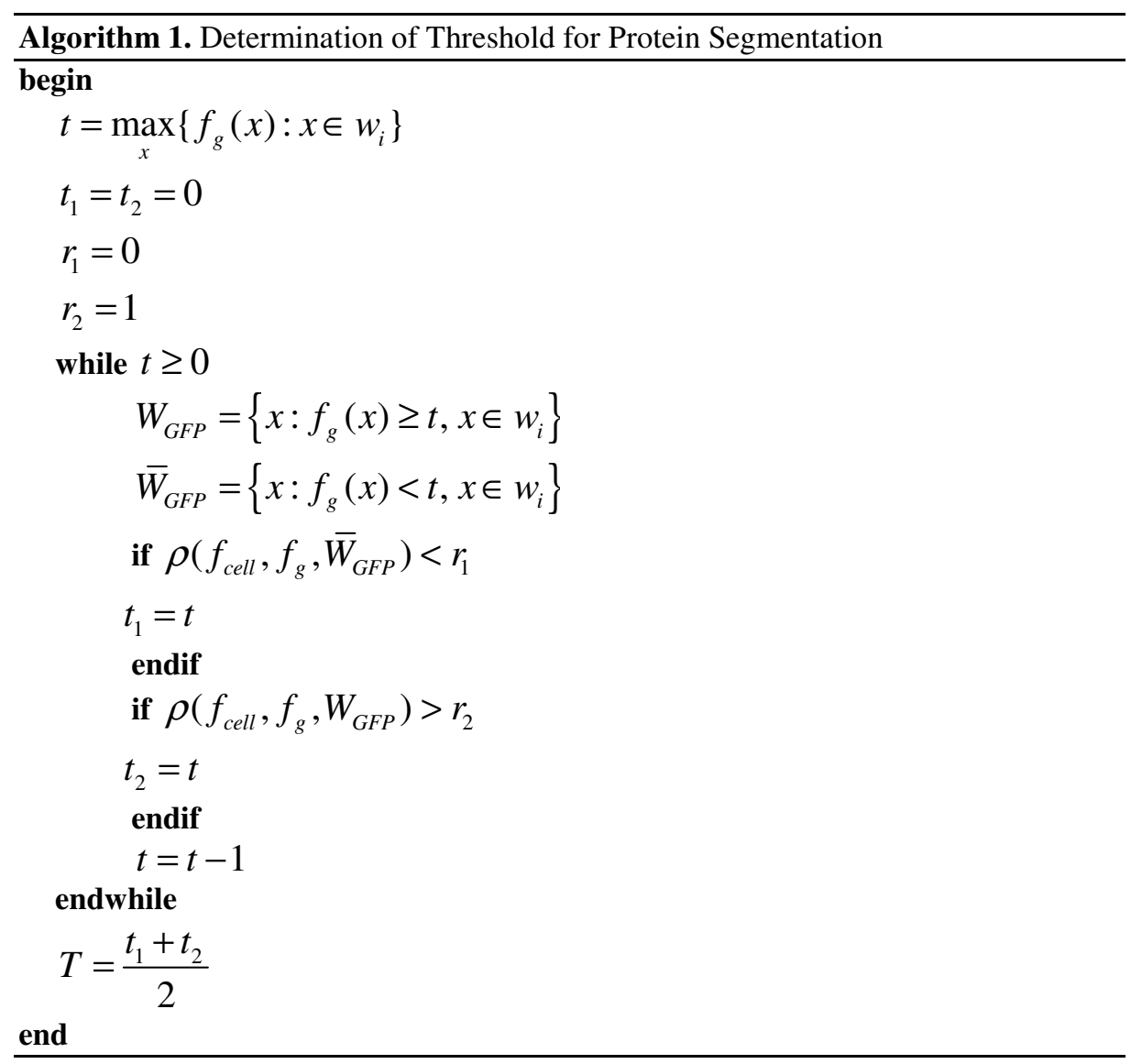

\subsection{Protein Localization Profiling}

Using a library $P$ of GFP-tagged cytoskeletal protein constructs, we compute the colocalization values of the protein in the five subcellular compartments. For given protein $p \in P$, the colocalization profile is $\operatorname{Col}(p)=\{\operatorname{Coloc}(c, p)\}$, where $c=\{$ nucleus, cytoplasm,actin,membrane, cytosol $\}$, we then cluster those protein profiles in order to find the functional proteins. 


\section{Experiments and Results}

\subsection{Sample Preparation}

Eighty-nine Invitrogen GFP-tagged cytoskeletal protein constructs were transfected to Hela cells in two 96-well plates ( 2 wells per construct, some constructs are duplicated). Each well has about 10,000 cells before transfection. Lipofectamine2000 transfections were taken to each well with constructs concentration 10ng/ul. Then the cells are fixed and stained with Hoechst33342 (nuclei) and Texas red phalloidin (Actin).

\subsection{Imaging}

Imaging of transfected cells was performed by Cellomics vHCS: Scan V Target Activation application system with $20 \mathrm{X}$ magnification. For each image sample there were 96 wells containing 48 constructs transfection results; and for each well there were 40 fields being scanned. Thus, the number of images in this dataset is about 7680. For each image, there are three fluorescent channels: blue (Hoechst33342) staining nuclei, red (Texas red - phalloidin) staining actin, and green (GFP) staining the particular protein.

\subsection{Image Processing}

After the high-content imaging, the images are analyzed with the computational frame work described in Methods section, involving individual cell segmentation, Intensity transformation of subcellular compartments, protein segmentation based on correlation coefficients, and colocalization quantification of proteins in subcellular components. For cell segmentation, a multi-resolution segmentation technique provided by Definiens Developer was used [18]. In the intensity transformation step, the fitting parameters for the exponential function are: $t_{m}=1.054, t_{m}^{\prime}=1.53$, $t_{m}^{\prime \prime}=2.241$.

A colocalization matrix is generated with the dimension of 89 proteins $\times 5$ subcellular compartments $\times$ the number of cells transfected with each protein. The colocalization matrix went through post data analysis to generate final conclusions.

\subsection{Cell Selection}

Before clustering the proteins, we apply a cell selection procedure based on nucleus area to delete part of the under-segmented cells as the nucleus area should have little variance in normal cells. The histogram of Nucleus Area feature is represented as a two peak curve, one peak is relatively smaller than the other. From biology we know that the higher peak shows the population of the normal nucleus and the smaller peak appears at the position where the area is twice of the normal nucleus area, showing the under-segmented two-connected nuclei. A single Gaussian-fit is applied to the histogram of nucleus area and the interval which contains $90 \%$ of the values from the fitting distribution was computed. The interval is [302,997]. Although the interval still contains some under-segmented nuclei, it successfully removes many of the misssegmented cells. 
In the current image dataset, the low transfection efficiency largely affects the final results of the colocalization analysis: a large number of cells are not transfected pulling down the total sample number; while the over-expressed ones show abnormal morphology and consequently abnormal colocalization results. After the transfection, the cytoskeletal proteins will first head to their normal subcellular locations or their functional locations. But in the over-expressed situation, more and more cytoskeletal proteins are generated and run everywhere inside the cell, which makes plenty of noise and reduces the significance of the functional locations of the particular cytoskeletal protein. Thus, we perform a GFP-intensity analysis to find the optimized intensity interval to eliminate abnormal-transfected cells.

In the GFP intensity analysis, the transfected cells are clustered into several GFPintensity groups. By computing the colocalization values for each GFP intensity group, the colocalization trends along with the increasing GFP intensities are investigated and we concluded that the colocalization values do change greatly with the increasing GFP intensity. In the high GFP intensity intervals (greater than 50), we could find that the colocalization values in the Nucleus compartment increased, indicating that the over-expressed cells could round up, as dead cells or toxic cells, which been proved by observation. This phenomenon affects the overall colocalization results, especially the constructs with low transfection efficiency. To keep the particular colocalization pattern as well as to avoid noise, the intensity interval $[20,30]$ seems to be a good choice for the cell selection.

\subsection{Colocalization Indexing}

In order to provide a standard comparison between subcellular compartments, we perform k-means clustering separately on all 5 colocalization values. The sums of squared distances are examined to determine the best number of clusters. For each $\mathrm{K}$, the K-means clustering is replicated 100 times to mitigate the effects of different initial conditions. Three is decided as the number of clusters as most of the colocalization values show inflection on it. The cluster labels from 1 to 3 are assigned to each protein to represent its colocalization degree for a specific subcellular compartment.

\subsection{Protein Clustering}

K-means clustering is performed again based on the cluster label of each protein for further protein classification. The sums of squared distances are examined to determine the best number of clusters. For each $\mathrm{K}$, the $\mathrm{K}$-means clustering is replicated 100 times to mitigate the effects from different initial conditions. Four is chosen as the number of the clusters and all the proteins are clustered into four clusters (Table 1): cluster $1-21$ proteins with equally distributions within cells; cluster $2-21$ proteins with high colocalization in plasma membrane; cluster $3-34$ proteins with high colocalization in actin and cytosol; and cluster $4-13$ proteins which are toxic to cells leading to cell round up. In Table 2 all proteins in each cluster are listed. As cytoskeletal proteins dynamically function within cells, the colocalization profiles will provide a distribution ratio among the subcellular compartments rather than one specific compartment. Although the exact functions of 
most of proteins remain unclear, the results can be validated with literature research. It is noticeable that some proteins in the same protein family are clustered together with similar colocalization profiles, such as TAGLN and TAGLN2, ITGB1and ITGB2, MYO3A and MYO1A, etc. Another validation is the comparison of reported functions of proteins and colocalization profiles. For example, cluster 3 shows high colocalization in actin compartment, and 22 of 34 proteins in this cluster are reported as having relative functions with the actin network.

Table 1. Colocalization of Protein Clusters

\begin{tabular}{c|cccccc}
\hline & Nucleus & CytoP & CytoS & Actin & PM & $\begin{array}{c}\text { protein } \\
\text { No. }\end{array}$ \\
\hline Cluster & $52.42 \%$ & $47.58 \%$ & $16.26 \%$ & $49.40 \%$ & $14.37 \%$ & 21 \\
$\mathbf{1}$ & $\pm 4.54 \%$ & $\pm 4.54 \%$ & $\pm 3.31 \%$ & $\pm 5.41 \%$ & $\pm 5.81 \%$ & \\
Cluster & $56.63 \%$ & $43.37 \%$ & $7.85 \%$ & $43.96 \%$ & $37.60 \%$ & 21 \\
$\mathbf{2}$ & $\pm 8.48 \%$ & $\pm 8.48 \%$ & $\pm 2.66 \%$ & $\pm 4.66 \%$ & $\pm 4.18 \%$ & 34 \\
Cluster & $34.70 \%$ & $65.30 \%$ & $18.62 \%$ & $53.23 \%$ & $20.54 \%$ & 13 \\
$\mathbf{3}$ & $\pm 6.08 \%$ & $\pm 6.08 \%$ & $\pm 3.36 \%$ & $\pm 7.53 \%$ & $\pm 5.50 \%$ & $15.57 \%$ \\
Cluster & $75.72 \%$ & $24.28 \%$ & $6.81 \%$ & $45.99 \%$ & $\pm 7.27 \%$ & \\
$\mathbf{4}$ & $\pm 9.07 \%$ & $\pm 9.07 \%$ & $\pm 3.07 \%$ & $\pm 13.04 \%$ & \pm & \\
\hline
\end{tabular}

Comparing the proteins within the same cluster and in different clusters, proteins with similar colocalization profiles are considered to have similar functions. For example, a set of proteins with unclear function such as filamin A interacting protein 1 (FILIP1) and tropomyosin 1 (TPM1), are seen to have similar profiles showing significantly high colocalization values in plasma membrane (cluster 2) together with other proteins having related functions with plasma membrane such as integrin beta 1 (ITGB1), integrin beta 2 (ITGB2), and villin 2 (VIL2).

Table 2. Proteins in Protein Clusters

\begin{tabular}{|c|c|c|}
\hline & protein No. & Protein Brief Name \\
\hline Cluster1 & 21 & $\begin{array}{l}\text { CORO2B,PDLIM3,DNM2,TAGLN,TNS,TEKT3,PTK9,PLS1,JAMIP2, } \\
\text { ATP1B3,VAMP4,PXN,MSN,ADRM1,MRLC2,TAGLN2,ARPC5,VIM, } \\
\text { NINJ2,PFN2,PARVA }\end{array}$ \\
\hline Cluster2 & 21 & $\begin{array}{l}\text { TUBA6,TGOLN2,CORO1B,ATP6V1C1,TAGLN3,VIL2,TUBD1, } \\
\text { TUBGCP3,PARVG,RDX,CAPZA3,ACTG1,PTK9,TPM1,ITGB1,ITGB2, } \\
\text { ADAM15,ARHGEF6,EIF2C1,WASF2,FILIP1 }\end{array}$ \\
\hline Cluster3 & 34 & $\begin{array}{l}\text { DCAMKL1,WASPIP,KLHDC9,CTTN,VIL1,ACTB,WASL,ZYX,CDC42, } \\
\text { CFL1,EVL,TUBE1,CLIP3,DSTN,PAK4,VASP,LPXN,KIF2C,ITGB7, } \\
\text { PLS3,ARPC1B,TUBG1,GTSE1,ACTN2,MYO3A,ACTN1,ARP11,CNN3, } \\
\text { LCP1,TPM2,MYO1A,ANLN,FSCN1,KRT8 }\end{array}$ \\
\hline Cluster4 & 13 & $\begin{array}{l}\text { WAS,ATP5G2,ITGB3BP,FSCN3,GJB2,TUBA1B,KPTN,TEKT1,TUBB2, } \\
\text { KAT5,WASF3,DCTN1,CAV3 }\end{array}$ \\
\hline
\end{tabular}




\section{Conclusions}

In this work, we developed a computational framework and optimized every step in the framework to quantify the subcellular localization of cytoskeletal proteins with a single colocalization measurement. The framework is applied on a two-dimensional image set, containing around 8000 images of cells transfected with 89 cytoskeletal protein constructs. The subcellular localizations of those cytoskeletal proteins are quantified and localization patterns are investigated to provide references in investigation of protein functions. Proteins with unknown functions can be investigated by comparing with colocalization profiles generated in a cytoskeletal protein library.

For image-based subcellular localization quantification, two-dimensional analysis is not sufficient. In future work, the whole framework will be transferred to the threedimensional domain. The quantification of subcellular localization on threedimensional space will provide more accurate results. The quantification of subcellular localization will greatly benefit the investigation of functions of cytoskeletal proteins.

Acknowledgments. This work was partly supported by Computation and Systems Biology programme of the Singapore-MIT Alliance.

\section{References}

1. Khurana, S., Bittar, E.: Aspects of the cytoskeleton. Elsevier Press, Amsterdam (2006)

2. Bray, D.: Cell Movements: From Molecules to Motility, 2nd edn. Taylor \& Francis Press, Abington (2001)

3. Hu, Y., Murphy, R.F.: Automated interpretation of subcellular patterns from immunofluorescence microscopy. Immunol Methods 290, 93-105 (2004)

4. Huang, M.R.F.: Boosting accuracy of automated classification of fluorescence microscope images for location proteomics. BMC Bioinformatics 5, 78-96 (2004)

5. Boland, M.V., Murphy, R.F.: A Neural Network Classifier Capable of Recognizing the Patterns of all Major Subcellular Structures in Fluorescence Microscope Images of HeLa Cells. Bioinformatics 17, 1213-1223 (2001)

6. Chen, X., Murphy, R.F.: Objective clustering of proteins based on subcellular location patterns. Journal of Biomedicine and Biotechnology, 87-95 (2005)

7. Hamilton, N., Pantelic, R., Hanson, K., Teasdale, R.: Fast automated cell phenotype image classification. BMC Bioinformatics 8, 110 (2007)

8. Chen, S.-C., Zhao, T., Gordon, G.J., Murphy, R.F.: Automated Image Analysis of Protein Localization in Budding Yeast. Bioinformatics 23, i66-i71 (2007)

9. Bolte, S., Cordelieres, F.P.: A guided tour into subcellular colocalization analysis in light microscopy. Journal of Microscopy 224(3), 213-232 (2006)

10. Manders, E.M., Stap, J., Brakenhoff, G.J., Driel, R., Aten, J.A.: Dynamics of threedimensional replication patterns during the S-phase, analysed by double labelling of DNA and confocal microscopy. J. Cell Sci. 103, 857-862 (1992)

11. Costes, S.V., Daelemans, D., Cho, E.H., Dobbin, Z., Pavlakis, G., Lockett, S.: Automatic and quantitative measurement of protein-protein colocalization in live cells. Biophys. J. 86, 3993-4003 (2004) 
12. Li, Q., Lau, A., Morris, T.J., Guo, L., Fordyce, C.B., Stanley, E.F.: A Syntaxin 1, Galphao, and N-type calcium channel complex at a presynaptic nerve terminal: analysis by quantitative immunocolocalization. J. Neurosci. 24, 4070-4081 (2004)

13. Boutte, Y., Crosnier, M.T., Carraro, N., Traas, J., Jeunemaitre, B.S.: Immuno cytochemistry of the plasma membrane recycling pathway and cell polarity in plants: studies on PIN proteins. J. Cell Sci. 113, 1255-1265 (2006)

14. Jaskolskia, F., Mullea, C., Manzonib, O.J.: An automated method to quantify and visualize colocalized fluorescent signals. J. Neurosci. Meth. 146, 42-49 (2005)

15. Cheng, J.R., Rajapakse, J.C.: Segmentation of clustered Nuclei with Shape Markers and Marking Function. IEEE Transactions on Biomedical Engineering 56(3), 741-748 (2009)

16. Yu, W., Lee, H.K., Hariharan, S., Bu, W., Ahmed, S.: Quantitative neurite outgrowth measurement based on image segmentation with topological dependence. Cytometry Part A 75A, 289-297 (2009)

17. McMaster Biophotonics Facility, http: / / www.macbiophotonics.ca/PDF/MBF_colocalisation.pdf

18. Baatz, M., Schäpe, A.: Multiresolution Segmentation -an optimization approach for high quality multi-scale image segmentation. In: Strobl, J., Blaschke, T., Griesebner, G. (eds.) Angewandte Geographische Informationsverarbeitung XII, pp. 12-23. Wichmann, Heidelberg (2000) 